\title{
Doing The Right Thing (and Figuring Out What That Is)
}

\author{
Barry M. Farr, MD, MSc
}

\begin{abstract}
How many times can a man turn his head, and pretend that he just doesn't see?
...Yes, and how many deaths will it take, till he knows that too many people have died?
\end{abstract}

Bob Dylan, "Blowing in the Wind"

When I was an impressionable freshman medical student, a senior clinician regularly showed up to educate and inspire the class with clinical correlations, holding us spellbound and ending each lecture with the same inscrutable advice, "Always do the right thing." On one hand, this seemed obvious, but on the other perplexing, because, as first-year students, we had no idea what "the right thing" might be. Years later, I noticed the same phrase in an episode of the television program $M^{*} A^{*} S^{*} H$ when Hawkeye received an award "for doing the right thing," and Frank Burns whined to Margaret, "How was I to know what the right thing was?"

Antibiotic-resistant infections are important causes of patient suffering and death. This started in the mid-1940s, right after penicillin began producing "miracle" cures. Sixty years later, it's still happening, and recent secular trend data suggest that the pattern has only gotten worse over the last few decades, despite multiple new antibiotics having been added to the physician's pharmaceutical armamentarium and infection control programs having been created three and a half decades ago primarily to control this problem. In most US hospitals, there has been little evidence of control; the incidence of methicillin-resistant Staphylococcus aureus (MRSA) infection increased 32-fold in the Centers for Disease Control and Prevention's (CDC) National Nosocomial Infections Surveillance system (NNIS) hospitals between 1976 and 2003. ${ }^{1}$ NNIS data also showed that the incidence of MRSA infection increased at roughly similar rates in small, medium, and large hospitals (Figure 1) and that MRSA infection rates in intensive care units (ICUs) usually paralleled and exceeded hospital-wide MRSA infection rates by several percentage points (Figure 2). In 2003, MRSA accounted for more than $64 \%$ of nosocomial S. aureus infections in NNIS hospital ICUs, ${ }^{1}$ but in some US hospitals it accounted for 70\%-90\%. MRSA and vancomycinresistant Enterococcus (VRE) infections have tended to be significantly more costly than comparable infections due to antibiotic-susceptible strains of the same species, ${ }^{2-8}$ and 4 recent meta-analyses of many previous studies concluded that they also were significantly more deadly. ${ }^{9-12}$

Two articles in this issue of the journal demonstrate that nosocomial MRSA infection can be controlled by active detection and isolation of all contagious patients, ${ }^{13,14}$ the same approach that has worked for containing other important nosocomial pathogens, such as those that cause tuberculosis, smallpox, and severe acute respiratory syndrome. ${ }^{15-18}$ Hospitals in Kfar Saba, Israel, ${ }^{13}$ and in Denver, Colorado, ${ }^{14}$ where MRSA had been endemic for years and rates of MRSA infection were still increasing, as in NNIS hospitals, ${ }^{1}$ despite use of standard precautions and isolation of patients known from clinical cultures to have MRSA, chose to start taking a more proactive approach to the problem. For both hospitals, this involved a program of performing active surveillance culture to identify colonized patients and then place them under contact isolation precautions, as recommended by a recent guideline from the Society for Healthcare Epidemiology of America. ${ }^{19}$ For Clancy and colleagues in Denver, this meant starting a program, and for Shitrit and colleagues in Kfar Saba, it meant enlarging an existing program 3-fold and transforming it from a reactive program triggered by detection of MRSA in clinical cultures to a proactive one that routinely screened patients at high risk for MRSA colonization or infection. Clancy et al. ${ }^{14}$ used only nasal cultures and intervened only in 2 ICUs, whereas Shitrit et al. ${ }^{13}$ used cultures of nose, perineum, dermatitis, and wound specimens, as well as sputum samples from intubated patients, and intervened throughout the hospital. Shitrit et al. ${ }^{13}$ performed cultures at admission for all high-risk patients and performed cultures at admission and periodically throughout the hospital stay for patients in high-risk units, which included ICUs and a long-term care unit for rehabilitative and skilled nursing care. Shitrit et al. ${ }^{13}$ also gave each colonized patient topical eradication therapy for 5 days. Both Shitrit et al. ${ }^{13}$ and Clancy et al. ${ }^{14}$ report significant reductions in the incidence of MRSA bacteremia over 18 and 15 months, respectively, and Clancy

Professor Emeritus, Department of Medicine, University of Virginia Health System, Charlottesville, Virginia.

Received August 28, 2006; accepted August 28, 2006; electronically published September 21, 2006.

Infect Control Hosp Epidemiol 2006; 27:999-1003

(C) 2006 by The Society for Healthcare Epidemiology of America. All rights reserved. 0899-823X/2006/2710-0001\$15.00. 


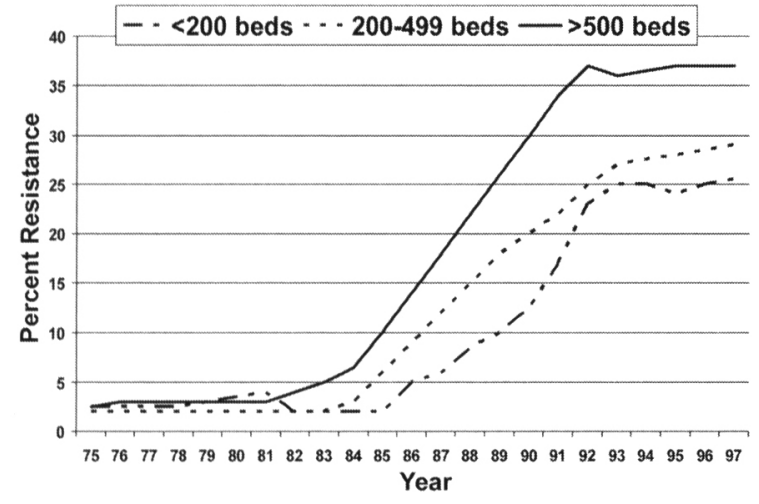

FIGURE 1. Prevalence of methicillin-resistant Staphylococcus aureus isolates causing nosocomial infections at National Nosocomial Infections Surveillance system (NNIS) hospitals between 1975 and 1997, according to the size of the hospitals. Data from the NNIS system, 1999.

et al. ${ }^{14}$ add that the reduction at their hospital had continued for another 11 months. These studies show that endemic MRSA infection in high-risk ICU settings can be controlled, confirming the results of many previous studies. ${ }^{20,21} \mathrm{Clancy}$ et al. ${ }^{14}$ report that control measures succeeded despite the apparent spread of staphylococcal cassette chromosome mec (SCCmec) type IV MRSA strains in the community resulting in community-acquired infections requiring hospitalization. This confirms observations that active detection and isolation of MRSA-colonized patients continues to control MRSA in healthcare facilities in areas where SCCmec type IV strains are spreading in the community. ${ }^{22,23}$ Clancy et al. ${ }^{14}$ also add a cost-benefit analysis showing that active detection and isolation of MRSA-colonized patients saved the hospital money, confirming the results of at least 13 prior studies that have reported cost savings with this method of controlling MRSA or VRE. ${ }^{24-36}$

In a third study in this issue of the journal, Warren et al. ${ }^{37}$ report on the use of nasal surveillance cultures in a surgical ICU in St. Louis to determine the prevalence of and risk factors associated with MRSA colonization, in preparation for an effort to control transmission. Eight percent of patients were colonized on admission to the ICU. Hospital admission in the past year, stay in a hospital ward for more than $\mathbf{5}$ days before transfer to the ICU, chronic obstructive pulmonary disease, diabetes mellitus, and a history of MRSA colonization were each significant predictors of colonization. Ten percent of patients with nasal cultures negative for MRSA on admission who stayed in the ICU for more than 2 days became culture positive for MRSA by the time of discharge, including some patients with a prior history of MRSA colonization. This suggests that nasal culture alone likely failed to detect some patients who were colonized at the time of admission, as noted by the authors, perhaps because the sensitivity of nasal culture alone for detecting colonized patients is $78 \%$ -
$93 \%$, as shown in several prior studies. ${ }^{38-41}$ Risk factors for acquisition of MRSA included having a tracheostomy, presence of a decubitus ulcer, or receipt of enteral nutrition via nasoenteric tube, percutaneous tube, or both.

In a fourth study in this issue of the journal, Lai et al. ${ }^{42}$ report failure to control MRSA transmission (after adjustment for colonization pressure) but dramatic control of VRE transmission (12 newly recognized cases of VRE colonization or infection per 1,000 patient-days before the intervention, compared with 3 newly recognized cases per 1,000 patientdays after the intervention). However, unlike the results of Shitrit et al. ${ }^{13}$ and Clancy et al., ${ }^{14}$ which concur with those of 100 prior studies, ${ }^{20,21}$ the results reported by Lai et $\mathrm{al}^{42}$ namely, that mere provision of alcohol-based handrubs greatly reduced VRE colonization-are inconsistent with most available data. Probably at least 6,000 US hospitals have used standard precautions since the CDC began recommending them in 1996, and most have likely used alcohol-based handrubs since a hand hygiene guideline recommended their use for most healthcare worker hand hygiene in $2002,{ }^{43}$ but relatively few hospitals have reported achieving significant control of MRSA or VRE that way. Like most hospitals, the University of Virginia Hospital has not reported achieving significant control with that approach because it wasn't seen, despite the installation of alcohol handrub dispensers hospital-wide (several years before this was done at the hospital described by Lai et $\mathrm{al}^{42}$ ) and despite use of hand hygiene compliance monitoring and feedback, which was followed by increases in compliance to $60 \%-80 \%$ in many wards and ICUs. Simply making alcohol-based handrubs available, along with educational and motivational information, has not always been associated with improved hand hygiene compliance, ${ }^{44}$ including at the University of Virginia Hospital, ${ }^{45}$ even though it has tried this on multiple occasions over decades. Another Virginia hospital reported endemic VRE ${ }^{46}$ and later reported a significant increase in hand hygiene compliance with use of alcohol handrubs, ${ }^{47}$ but it has not published data

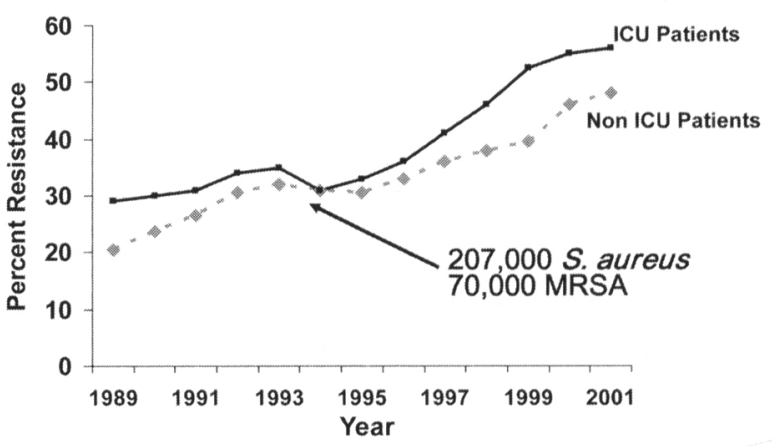

FIGURE 2. Prevalence of methicillin-resistant Staphylococcus aureus isolates causing nosocomial infections in intensive care unit (ICU) patients and non-ICU patients at National Nosocomial Infections Surveillance system (NNIS) hospitals between 1989 and 2001. Data from the NNIS system, 2002, courtesy S. Fridkin. 
showing that the improved compliance dramatically controlled VRE or MRSA transmission. Huang et al. ${ }^{48}$ reported a $75 \%$ reduction in the number of cases of MRSA bacteremia with use of active detection and isolation of all colonized patients in ICUs at Brigham and Women's Hospital (Boston, MA), but they reported no effect on the MRSA bacteremia rate after provision of alcohol handrubs and institution of a motivational campaign that succeeded in increasing hand hygiene compliance to $80 \%$.

There are other troubling things that raise questions about the credibility of the findings of Lai et $\mathrm{al}^{42}$ First, the brief alcohol handrub intervention ( 5 months) without compliance monitoring followed 8 years of reported VRE endemicity, which suggests VRE endemicity in the surrounding healthcare system as well. This makes such a dramatic, rapid reduction in the rate of VRE colonization seem improbable (in part because some patients in a healthcare system with endemic VRE who become culture positive in an ICU do so merely because of antibiotic therapy they receive in the ICU, having acquired VRE elsewhere in the system), and there was no update appended (as by Clancy et al. ${ }^{14}$ ) to say what happened in the intervening 5 years since the study was done. Second, the absolute reduction in newly recognized colonized patients between the 2 study periods was only 10 patients (ie, approximately 2 per month). If analyzed using cumulative incidence, there was no significant difference: 29 of 80 patients in the first period, compared with 19 of 79 patients in the second period (Yates corrected $\chi^{2}, 2.26 ; P=.13$; relative risk, $0.66)$. Univariate analysis using incidence-density data probably wouldn't have made much difference in these results, implying that the reported $75 \%$ reduction in the relative risk could only have occurred because of major changes in some other variable(s) entered into multivariable analysis. However, neither the Results nor the Methods section indicates that such an analysis was done for the incidence of VRE colonization, nor what variable(s) might have explained such a major change in results between univariate and multivariate analyses. With respect to MRSA, adjustment for prevalent cases of MRSA colonization or infection reportedly led to a different result from that expected on the basis of univariate analysis, but a similar statement was not made as an explanation for the reported VRE result, which also was unexpected on the basis of univariate analysis.

Third, in the Results section, Lai et al. ${ }^{42}$ say they achieved a "2.25-fold reduction in the incidence of VRE" (p. 1021). As mentioned above, it is not clear from the raw data provided that the rate actually was reduced from 12 to 3 cases per 1,000 patient-days, but, if so, this would represent a $75 \%$ reduction in the relative risk; a reduction to 0 would have represented a $100 \%$, or 1 -fold, reduction. It is not clear what the authors mean by a " 2.25 -fold reduction in the incidence." Fourth, the fact that the prevalence of VRE colonization or infection in the 2 study periods reportedly remained roughly the same (ie, $25 \%$ and $26 \%$, respectively) makes the reported dramatic reduction in VRE acquisition seem questionable. The lack of change in prevalence also would seem to imply that more patients with VRE had to be admitted, but Lai et al. ${ }^{42}$ write in the Discussion section that "this [finding] could not be explained by an increased incidence of VRE cases among patients admitted from outside the institution, because there was no significant increase in the number of patients with VRE colonization or infection admitted to the medical ICU.... The decrease was seen despite a higher prevalence of VRE cases after the alcohol hand antiseptic was made available" (p. 1022). This math doesn't seem to add up.

Fifth, in the Discussion section, Lai et al, ${ }^{42}$ seek to show consistency of their results with those of other studies. They write that "the benefit of improved infection control practices, including hand hygiene practices, in reducing nosocomial infection rates has been demonstrated" (p. 1022) and cite 2 articles, the first a study reporting that a relative $38 \%$ increase in hand hygiene compliance (ie, from $48 \%$ to $66 \%$ compliance) was temporally associated with a relative $57 \%$ decrease in cases of MRSA acquisition, ${ }^{49}$ and the second the recent hand hygiene guideline. ${ }^{43}$ However, the hand hygiene intervention study they cite ${ }^{49}$ was likely confounded by simultaneous implementation of a large program of active surveillance culture and implementation of contact precautions for all colonized patients, as detailed in another publication by the same investigators, ${ }^{50}$ and the hand hygiene guideline contained no data showing profound, prolonged control of MRSA and VRE with hand hygiene alone. ${ }^{43}$

A recently published essay suggested that controlling MRSA is "simple," declaring that hand hygiene will result in "an immediate and profound reduction," "51(p122) but cited no data showing this to be true, perhaps because of the paucity of such data. H. L. Mencken said "There is always an easy solution to every human problem-neat, plausible, and wrong." Besides the lack of compelling data consistently showing this, another problem with assuming that hand hygiene alone could control this problem is that examining an MRSA- or VRE-colonized, nonisolated patient frequently results in contamination of a clinician's clothes and personal or medical equipment, ${ }^{52,53}$ the Achilles' heel of even "enhanced" standard precautions, which ignore this portable contagion. The essay blamed the unwashed hands of American clinicians for decades of uncontrolled MRSA infection, ${ }^{51}$ suggesting that clinicians hadn't been educated and motivated properly, despite the fact that the $\mathrm{CDC}$ has recommended handwashing before and after every patient contact as part of Standard Precautions since 1996 and despite federal regulations mandating such measures, as well as annual retraining in infection control, for all healthcare workers in all US healthcare facilities.

American physicians and nurses are as good, knowledgeable, and caring as their counterparts in northern European countries and Western Australia, where nosocomial MRSA infections have been consistently and convincingly controlled to very low levels for decades using active detection and isolation (ie, a practicable system). ${ }^{23,54}$ That contrasts sharply with the much higher rates of nosocomial MRSA infection 
in other European nations and other Australian states that do not routinely use this approach, ${ }^{54,55}$ including some that have emphasized hand hygiene for years, apparently to no avail. The results of more than 100 studies reporting control of MRSA by means of active detection and isolation, ${ }^{20,21}$ including those by Shitrit et al. ${ }^{13}$ and Clancy et al. ${ }^{14}$ in this issue, corroborate these findings. This suggests that if blame were to be assigned for the American healthcare system's failure to control MRSA and VRE, which has resulted in hundreds of thousands of preventable nosocomial infections and more than 10,000 deaths each year, those responsible for (but never) recommending effective control measures might deserve a more prominent share.

Infection control professionals in US hospitals tend to follow CDC guidelines, which have never explicitly recommended routine performance of active surveillance culture to identify colonized patients and isolation of all such patients in order to control nosocomial MRSA infection, as has been done in multiple nations that have controlled it to exceedingly low levels for decades. It should have been obvious for many years to infection control professionals and to CDC officials that isolation of only the small fraction of MRSA-colonized patients identified on the basis of clinical cultures (as has been done by most US hospitals since 1983) and use of standard precautions (as has been done by most US hospitals since 1996) have failed miserably to control nosocomial MRSA and VRE infections. "How many deaths will it take till they know that too many people have died" and that these controllable infections should start being controlled?

Address correspondence to Barry M. Farr, MD, at bmf@virginia.edu.

\section{REFEREN CES}

1. Klevens R, Edwards J, Tenover F, McDonald L, Horan T, Gaynes R. National Nosocomial Infections Surveillance System. Changes in the epidemiology of methicillin-resistant Staphylococcus aureus in intensive care units in US hospitals, 1992-2003. Clin Infect Dis 2006; 42:389-391.

2. Abramson MA, Sexton DJ. Nosocomial methicillin-resistant and methicillin-susceptible Staphylococcus aureus primary bacteremia: at what costs? Infect Control Hosp Epidemiol 1999; 20:408-411.

3. Cheng AF, French GL. Methicillin-resistant Staphylococcus aureus bacteremia in Hong Kong. $J$ Hosp Infect 1988; 12:91-101.

4. Stone PW, Larson E, Kawar LN. A systematic audit or economic evidence linking nosocomial infections and infection control interventions: 19902000. Am J Infect Control 2002; 30:145-152.

5. Cosgrove SE, Qi Y, Kaye KS, Harbarth S, Karchmer AW, Carmeli Y. The impact of methicillin resistance in Staphylococcus aureus bacteremia on patient outcomes: mortality, length of stay, and hospital charges. Infect Control Hosp Epidemiol 2005; 26:166-174.

6. Reed SD, Friedman JY, Engemann JJ, et al.Costs and outcomes among hemodialysis-dependent patients with methicillin-resistant or methicillin-susceptible Staphylococcus aureus bacteremia. Infect Control Hosp Epidemiol 2005; 26:175-183.

7. McHugh CG, Riley LW. Risk factors and costs associated with methicillinresistant Staphylococcus aureus bloodstream infections.Infect Control Hosp Epidemiol 2004; 25:425-430.
8. Stosor V, Peterson L, Postelnick M, Noskin G. Enterococcus faecium bacteremia: does vancomycin resistance make a difference? Arch Intern Med $1998 ; 158: 522-527$.

9. Cosgrove SE, Sakoulas G, Perencevich EN, Schwaber MJ, Karchmer AW, Carmeli Y. Comparison of mortality associated with methicillin-resistant and methicillin-susceptible Staphylococcus aureus bactermia: a mclanetaanalysis. Clin Infect Dis 2003; 36:53-59.

10. Whitby M, McLaws ML, Berry G. Risk of death from methicillin-resistant Staphylococcus aureus bacteraemia: a meta-analysis. Med J Aust 2001; 175:264-267.

11. Salgado $C D$, Farr BM. Outcomes associated with vancomycin resistant enterococci: a meta-analysis. Infect Control Hosp Epidemiol 2003; 24: 690-698.

12. Diaz Granados CA, Zimmer SM, Klein M, Jernigan JA. Comparison of mortality associated with vancomycin-resistant and vancomycin-susceptible enterococcal bloodstream infections: a meta-analysis. Clin Infect Dis $2005 ; 41: 327-333$.

13. Shitrit P, Gottesman BS, Katzir M, Kilman A, Ben-Nissan Y, Chowers M. Active surveillance for methicillin-resistant Staphylococcus aureus (MRSA) decreases the incidence of MRSA bacteremia. Infect Control Hosp Epidemiol 2006; 27:1004-1008 (in this issue).

14. Clancy M, Graepler A, Wilson M, Douglas I, Johnson J, Price C. Active screening in high-risk units is an effective and cost-avoidant method to reduce the rate of methicillin-resistant Staphylococcus aureus infection in the hospital. Infect Control Hosp Epidemiol 2006; 27:1009-1017 (in this issue).

15. Sommer A. SARS: paradigm for an emerging pathogen in a global community. Paper presented at: 14th Annual Meeting of The Society for Healthcare Epidemiology of America; Philadelphia, PA; April 17, 2004.

16. Seto WH, Tsang D, Yung RW, et al., Advisors of Expert SARS Group of Hospital Authority. Effectiveness of precautions against droplets and contact in prevention of nosocomial transmission of severe acute respiratory syndrome (SARS). Lancet 2003; 361:1519-1520.

17. Lipsitch $\mathrm{M}$, Cohen $\mathrm{T}$, Cooper $\mathrm{B}$, et al. Transmission dynamics and control of severe acute respiratory syndrome. Science 2003; 300:1966-1970.

18. Beck-Sague CM, Dooley SW, Hutton MD, et al. Outbreak of multidrugresistant tuberculosis among persons with HIV infection in an urban hospital: transmission to staff and patients and control measures. JAMA $1992 ; 268: 1280-1286$.

19. Muto CA, Jernigan JA, Ostrowksy BE, et al. SHEA guideline for preventing nosocomial transmission of multidrug-resistant strains of Staphylococcus aureus and Enterococcus. Infect Control Hosp Epidemiol 2003; 24:362-386.

20. Farr BM. What to think if the results of the National Institutes of Health randomized trial of methicillin-resistant Staphylococcus aureus and vancomycin-resistant Enterococcus control measures are negative (and other advice to young epidemiologists): a review and an au revoirr. Infect Control Hosp Epidemiol 2006; 27:1096-1106 (in this issue).

21. Salgado CD, Farr BM. The importance of infection control in controlling antimicrobial-resistant pathogens. In: Jarvis WR, ed. Bennett and Brachman's Hospital Infections. 5th ed. Lippincott Williams \& Wilkins; Philadelphia (in press).

22. Urth T, Juul G, Skov R, Schonheyder HC. Spread of a methicillinresistant Staphylococcus aureus ST80-IV clone in a Danish community. Infect Control Hosp Epidemiol 2005; 26:144-149.

23. Dailey L, Coombs GW, O'Brien FG, et al. Methicillin-resistant Staphylococcus aureus, Western Australia. Emerg Infect Dis 2005; 11:1584-1590.

24. West TE, Guerry C, Hiott M, Morrow N, Ward K, Salgado CD. Effect of targeted surveillance for control of methicillin-resistant Staphylococcus aureus in a community hospital system. Infect Control Hosp Epidemiol 2006; 27:233-238.

25. Lucet J, Chevret S, Durand-Zaleski I, Chastang Cregnier B. Prevalence and risk factors for carriage of methicillin resistant Staphylococcus aureus at admission to the intensive care unit. Arch Intern Med 2003; 163:181-188.

26. Bjorholt I, Haglind E. Cost-savings achieved by eradication of epidemic 
methicillin-resistant Staphylococcus aureus (EMRSA)-16 from a large teaching hospital. Eur J Clin Microbiol Infect Dis 2004; 23:688-695.

27. Vriens M, Blok H, Fluit A, Troelstra A, van der Werken C, Verhoef J. Costs associated with a strict policy to eradicate methicillin-resistant Staphylococcus aureus in a Dutch University Medical Center: a 10-year survey. Eur J Clin Microbiol Infect Dis 2002; 21:782-786.

28. Karchmer TB, Durbin LJ, Simonton BM, Farr BM. Cost-effectiveness of active surveillance cultures and contact/droplet precautions for control of methicillin-resistant Staphylococcus aureus. J Hosp Infect 2002; 51:126-132.

29. Papia G, Louie M, Tralla A, Johnson C, Collins V, Simor AE. Screening high-risk patients for methicillin-resistant Staphylococcus aureus on admission to the hospital: is it cost effective? Infect Control Hosp Epidemiol 1999; 20:473-477.

30. Chaix C, Durand-Zaleski I, Alberti C, Brun-Buisson C. Control of endemic methicillin-resistant Staphylococcus aureus: a cost-benefit analysis in an intensive care unit. JAMA 1999; 282:1745-1751.

31. Jernigan JA, Clemence MA, Stott GA, et al. Control of methicillin resistant Staphylococcus aureus at a university hospital: one decade later. Infect Control Hosp Epidemiol 1995; 16:686-696.

32. Muto CA, Giannetta ET, Durbin LJ, Simonton BM, Farr BM. Cost-effectiveness of perirectal surveillance cultures for controlling vancomycinresistant Enterococcus. Infect Control Hosp Epidemiol 2002; 23:429-435.

33. Montecalvo MA, Jarvis WR, Uman J, et al. Costs and savings associated with infection control measures that reduced transmission of vancomycin-resistant enterococci in an endemic setting. Infect Control Hosp Epidemiol 2001; 22:437-442.

34. Bronstein M, Kaye K, Sexton D. Gown utilization as a measure of cost of methicillin-resistant Staphylococcus aureus (MRSA) screening. In: Program and abstracts of the 12th Annual Meeting of the Society for Healthcare Epidemiology of America; April 6-9, 2002; Salt Lake City, UT. Abstract 47.

35. Calfee DP, Farr BM. Infection control and cost control in the era of managed care. Infect Control Hosp Epidemiol 2002; 23:407-410.

36. Peterson L. Lessons to be shared in successful reductions of infections. Paper presented at: Illinois Quality Leadership Conference-Focusing on Reducing and Eliminating Infections. Naperville, IL: Illinois Hospital Association and Metropolitan Chicago Healthcare Council; May 24, 2006.

37. Warren DK, Guth RM, Coopersmith CM, Merz LR, Zack JE, Fraser VJ, Epidemiology of methicillin-resistant Staphylococcus aureus colonization in a surgical intensive care unit. Infect Control Hosp Epidemiol 2006; 27: 1032-1040 (in this issue).

38. Girou E, Pujade G, Legrand P, Cizeau F, Brun-Buisson C. Selective screening of carriers for control of methicillin-resistant Staphylococcus aureus (MRSA) in high-risk hospital areas with a high level of endemic MRSA. Clin Infect Dis 1998; 27:543-550.

39. Lucet IC, Chevret S, Durand-Zaleski I, Chastang C, Regnier B, Multicenter Study Group. Prevalence and risk factors for carriage of methicillin-resistant Staphylococcus aureus at admission to the intensive care unit: results of a multicenter study. Arch Intern Med 2003; 163:181-188.

40. Coello R, Jimenez J, Garcia M, et al. Prospective study of infection, colonization and carriage of methicillin-resistant Staphylococcus aureus in an outbreak affecting 990 patients. Eur J Clin Microbiol Infect Dis 1994; 13:74-81.

41. Sanford MD, Widmer AF, Bale MI, Jones RN, Wenzel RP. Efficient de- tection and long-term persistence of the carriage of methicillin-resistant Staphylococcus aureus. Clin Infect Dis 1994; 19:1123-1128.

42. Lai KK, Fontecchio S, Melvin Z, Baker SP. Impact of alcohol-based, waterless hand antiseptic on the incidence of infection and colonization with methicillin-resistant Staphylococcus aureus and vancomycin-resistant enterococci. Infect Control Hosp Epidemiol 2006; 27:1018-1024 (in this issue).

43. Boyce JM, Pittet D, Healthcare Infection Control Practices Advisory Committee, Society for Healthcare Epidemiology of America, Association for Professionals in Infection Control, Infectious Diseases Society of America Hand Hygiene Task Force. Guideline for hand hygiene in healthcare settings: recommendations of the Healthcare Infection Control Practices Advisory Committee and the HICPAC/SHEA/APIC/IDSA Hand Hygiene Task Force. MMWR Recomm Rep 2002; 51(RR-16):1-56.

44. Muller A, Denizot V, Mouillet S, et al. Lack of correlation between consumption of alcohol-based solutions and adherence to guidelines for hand hygiene. J Hosp Infect 2005; 59:163-164.

45. Muto CA, Sistrom MG, Farr BM. Hand hygiene rates unaffected by installation of dispensers of a rapidly acting hand antiseptic. Am J Infect Control 2000; 28:273-276.

46. Bischoff WE, Reynolds TM, Hall GO, Wenzel RP, Edmond MB. Molecular epidemiology of vancomycin-resistant Enterococcus faecium in a large urban hospital over a 5-year period. J Clin Microbiol 1999; 37:3912-3916.

47. Bischoff WE, Reynolds TM, Sessler CN, Edmond MB, Wenzel RP. Handwashing compliance by health care workers: the impact of introducing an accessible, alcohol-based hand antiseptic. Arch Intern Med 2000; 160: 1017-1021.

48. Huang S, Yokoe D, Rego V, et al. Impact of ICU surveillance for MRSA on bacteremia. In: Program and abstracts of the 43rd Annual Meeting of the Infectious Diseases Society of America; Oct 6-9, 2005; San Francisco, CA. Abstract 1074.

49. Pittet D, Hugonnet S, Harbarth S, et al. Effectiveness of a hospital-wide programme to improve compliance with hand hygiene. Infection Control Programme [published correction appears in Lancet 2000; 356:2196]. Lancet 2000; 356:1307-1312.

50. Harbarth S, Martin Y, Rohner P, Henry N, Auckenthaler R, Pittet D. Effect of delayed infection control measures on a hospital outbreak of methicillin-resistant Staphylococcus aureus. J Hosp Infect 2000; 46:43-49.

51. Goldmann D. System failure versus personal accountability-the case for clean hands. $N$ Engl J Med 2006; 355:121-123.

52. Zachary KC, Bayne PS, Morrison VJ, Ford DS, Silver LC, Hooper DC. Contamination of gowns, gloves, and stethoscopes with vancomycinresistant enterococci. Infect Control Hosp Epidemiol 2001; 22:560-564.

53. Boyce J, Chenevert C. Isolation gowns prevent health care workers (HCWs) from contaminating their clothing, and possibly their hands, with methicillin-resistant Staphylococcus aureus (MRSA) and resistant enterococci. In: Program and abstracts of the 8th Annual Meeting of the Society for Healthcare Epidemiology of America; Orlando, FL; 1998. Abstract S74.

54. Tiemersma EW, Bronzwaer SL, Lyytikainen O, et al. Methicillin-resistant Staphylococcus aureus in Europe, 1999-2002. Emerg Infect Dis 2004; 10: 1627-1634.

55. Boyce JM, Cookson B, Christiansen K, et al. Methicillin-resistant Staphylococcus aureus. Lancet Infect Dis 2005; 5:653-663. 